\title{
Chronic prolymphocytoid leukaemia with an unusual immature immunophenotype
}

\author{
M Lima, J Coutinho, A Orfão, A L Macedo, J F San Miguel, B Justiça
}

\begin{abstract}
A case of a 58 year old woman with a chronic lymphoproliferative disorder of unusual clinical presentation, disease course, and immunophenotype is presented. At diagnosis she had severe anaemia, moderate lymphocytosis with some cells having prolymphocytoid features and a normal platelet count. A clinical examination yielded negative results. Only anaemia related symptoms were found and the patient became blood transfusion dependent. Both the lymphocytosis and the proportion of prolymphocytoid cells rose insidiously and thrombocytopenia developed later during the course of the disease. Three years later, the patient had a white cell count of $269 \times 10^{9} / 1$ almost exclusively of prolymphocytoid cells and the bone marrow was diffusely infiltrated. She was refractory to chemotherapy and the anaemia did not improve after treatment with cyclosporine. Lymphoid cells were positive for cytoplasmatic CD3, HLA-Dr, CD34, CD38, CD7, CD56, CD13, CD33 and CD65. Membrane $\alpha \beta$ and $\gamma \delta$ T cell receptors (TCRs) were not expressed and the $\beta$ chain TCR gene was in germline configuration. Other membrane $T, B$, natural killer, and myelomonocytic markers were negative. Karyotype analysis was tried several times but metaphases were not obtained, even after stimulation with $T$ cell mitogens.
\end{abstract}

( Clin Pathol 1994;47:461-463)

Several attempts have been made to classify both acute and chronic leukaemias, most of them based on cell morphology. ${ }^{12}$ Nevertheless, immunophenotyping has helped considerably in the diagnosis of these hematological disorders, ${ }^{3}$ an immature phenotype usually being associated with an acute disease course. We describe a chronic lymphoproliferative disorder displaying an unusual immature immunophenotype. As far as we know no other case with these characteristics has been reported before.

Case report

A 58 year old white woman was admitted to hospital in January 1990 because of anaemia and lymphocytosis. Both had first been noted six months earlier when she consulted her physician because of fatigue, dyspnoea, and palpitations. During that time she was not given any treatment.

The blood picture at diagnosis was as follows: haemoglobin $55 \mathrm{~g} / \mathrm{l}$; platelet count $183 \times 10^{9} / 1$; white cell count $14.6 \times 10^{9} / 1$, with $88 \%$ lymphoid cells $(20 \%$ of them displaying a prolymphocytoid morphology), and $12 \%$ neutrophils. No splenomegaly, hepatomegaly, lymphadenopathy, skin lesions, or other abnormal findings were noted on clinical examination. Serological evidence for hepatitis $B$ virus, HIV, and Epstein-Barr virus infection was not found. A direct Coombs' test was negative.

A bone marrow aspirate showed the presence of a lymphoid infiltrate with $43 \%$ prolymphocytoid cells and $29 \%$ mature-looking lymphocytes. Sixteen per cent of the bone marrow cells were myeloid and $12 \%$ were erythroid elements with all maturation stages of these cell lineages being present. Lymphocyte immunophenotyping at that time was not helpful: bone marrow and peripheral blood lymphoid cells were positive for HLA-Dr and negative for CD19, CD20, CD10, surface immunoglobulin (sIg), cytoplasmatic $\mu$ chains (c $\mu$ ) CD2, CD 5, CD3, CD4, CD8, CD14, CD13 and CD71. Cytochemical data were not available (table). A peripheral blood karyotype analysis was not possible because no metaphases were obtained after a 24 and 48 h

Peripheral blood immunophenotype

\begin{tabular}{llll}
\hline & \multicolumn{3}{l}{ Positive cells (\%) } \\
\cline { 2 - 4 } & 1990 & 1991 & 1992 \\
\hline HLA-Dr & 48 & 64 & 74 \\
CCD3 & NT & NT & 98 \\
CD7 & NT & NT & 99 \\
CD13 & NT & 70 & 97 \\
CD15 & NT & NT & 15 \\
CD29 & NT & NT & 77 \\
CD33 & NT & 25 & 87 \\
CD34 & NT & NT & 98 \\
CD38 & NT & NT & 54 \\
CD45R0 & NT & NT & 86 \\
CD56 & NT & NT & 99 \\
CD65 & NT & NT & 35 \\
CD71 & 0 & NT & 99 \\
\hline
\end{tabular}

^ Less than $10 \%$ of the peripheral blood lymphoid cells were positive for the following markers: CD2, CD $3, \mathrm{CD} 4, \mathrm{CD} 5$, positive for the following markers: CD2, CD3, CD4, CD5, CD22, CD25, CD41a, CD45RA, CD57, TCR $a$, , TCR $\delta$, sIg, $\mathrm{c} \mu$. 
culture, both in the absence and in the presence of phytohaemaglutinin (PHA). Serum immunoglobulin concentrations were normal and monoclonal bands were not found.

In spite of the prolymphocytoid features of peripheral blood and bone marrow cells, prolymphocytic leukaemia (PLL) was excluded by negativity for $B$ and $T$ cell associated markers, absence of splenomegaly, and only moderately increased white cell counts.

During the course of the disease, the patient became blood transfusion dependent and the white cell count rose insidiously, with a variable but progressive rise in the percentage of cells displaying prolymphocytoid morphology.

By December 1990 the white cell count reached $95 \times 10^{9} / 1$, the platelet count was $154 \times 10^{9} / 1$, and haemoglobin was $50 \mathrm{~g} / 1$. Clinical examination remained negative and only symptoms related to anaemia were found. Treatment with chlorambucil and Prednisolone (four courses) was given without any obvious response.

In October 1991 the white cell count was $100 \times 10^{9} / 1$ and thrombocytopenia was noticed for the first time (platelets $50 \times 10^{9} / 1$ ). The bone marrow and peripheral blood immunophenotyping was repeated using the same panel of monoclonal antibodies as that used in December 1990, with similar results to those obtained for the first study, except for a variable proportion of cells reacting with both CD13 and CD33 myeloid associated markers.

In January 1992 the lymphocyte immunophenotype was evaluated with an expanded panel of monoclonal antibodies with a broad range of specificities. The main feature found at that time was the strong positivity for both CD7 and CD56. CD25 and CD45RA were negative, while CD29 was expressed on most

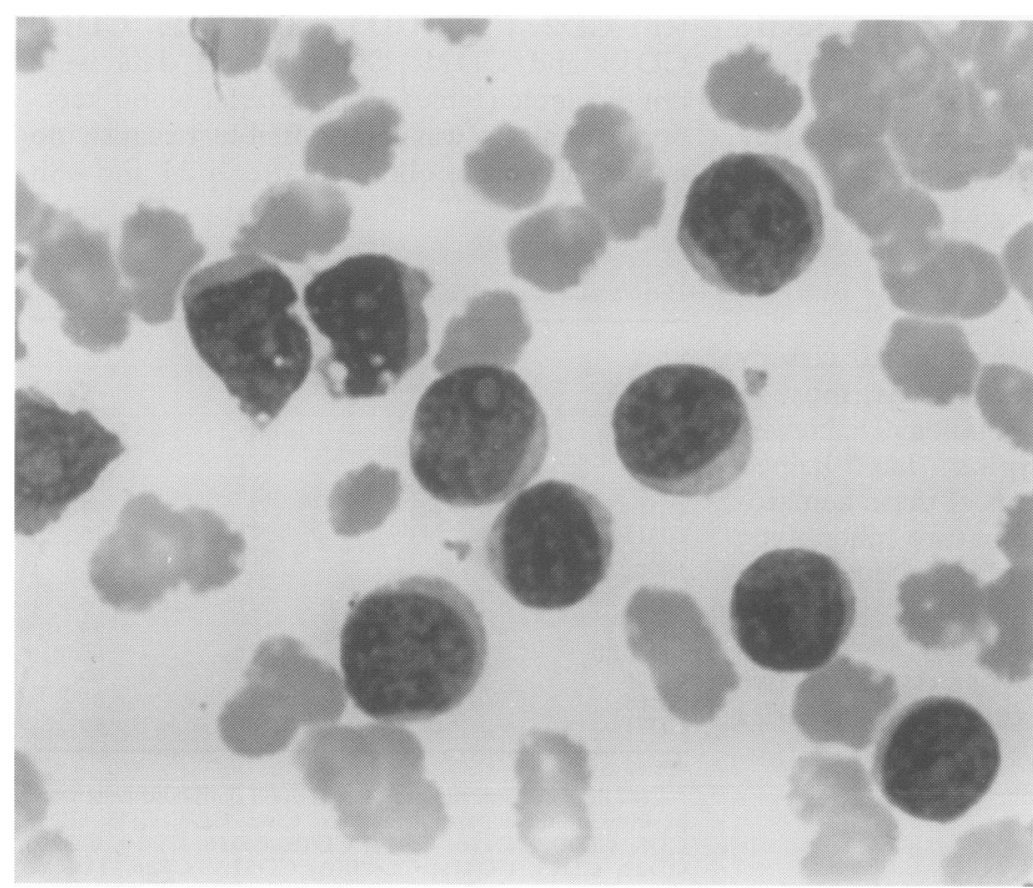

Peripheral blood film (Leishman's stain). lymphoid cells. Negativity for other T (CD2, CD5, CD3, CD4, CD8) and B (CD19, CD20, CD22, CD10) cell lineage associated markers was confirmed. Genetic analysis showed that $\beta$ chain TCR was in germline configuration.

Severe iron overload, due to multiple blood transfusions, progressively developed and the erythropoietin concentration was increased $(>200 \mathrm{mU} / \mathrm{ml})$. Cyclosporin treatment was therefore started in February 1992 for three months. Not only did the patient fail to respond to this treatment but she also tolerated it very poorly. By February 1992 the patient had become progressively and insidiously weaker, and experienced fatigue, anorexia, and weight loss.

By July 1992, the haematological picture was as follows: a white cell count of $250 \times 10^{9} / 1$; haemoglobin $50 \mathrm{~g} / \mathrm{l}$; and a platelet count of less than $10 \times 10^{9} / 1$. Both the peripheral blood smear and the bone marrow aspirate contained almost exclusively prolymphocytoid cells (figure) and a trephine biopsy specimen showed $70 \%$ haematopoietic cells, almost exclusively represented by lymphoid cells, described as "small lymphocytes with prominent irregular nucleoli". Acid phospatase was weakly positive with a nonpolar distribution and myeloperoxidase and Tdt were negative. Immunophenotyping studies showed reactivity with CD34, CD38, CD7, CD56 as well as with CD13, CD33, and CD65. Other B (CD19, CD20, CD10), T (CD2, CD5, CD3, CD4, CD8), natural killer (CD16, CD57, CD11b), and myelomonocytic associated markers (CD14, CD11b, CD15) were negative. Cytoplasmatic CD3 (cCD3) was assayed for the first time, and was strongly positive. Membrane $\alpha \beta$ and $\gamma \delta$ TCR were not expressed and the $\beta$ chain TCR gene was in germline configuration. Flow cytometric cell cycle analysis showed that peripheral blood lymphoid cells had a normal diploid DNA content with only $0 \cdot 1 \%$ being at $S$ phase (absolute $S$ phase white cell count of $\left.0 \cdot 25 \times 10^{9} / 1\right)$. Karyotype analysis was tried again but no metaphases were obtained. HTLV1 was negative.

In August 1992 combined chemotherapy with cyclophosphamide, vincristine, and prednisolone was started. After four courses the white cell count fell from 269 to $23 \times 10^{9} / 1$, but the haemoglobin concentration and platelet counts did not improve, and the patient remained profoundly anaemic and thrombocytopenic. She died in February 1993.

\section{Discussion}

In this report we have described a chronic lymphoproliferative disorder, with morphological evidence of a prolymphocytoid leukaemia and an unusual immunophenotype suggestive of immature $T$ cell proliferation. In spite of the morphological prolymphocytoid features, several data argue against T-PLL. The unusual presentation with anaemia was the main clinical and laboratory feature; the 
insidious clinical course during which the white cell count progressively increased as did the percentage of prolymphocytoid cells but during which neither spleen nor liver enlarged; and finally, the unusual immunophenotype. In fact, a high white cell count, multiorgan disease, and an aggressive clinical course with a median survival of $7 \cdot 5$ months are characteristic features of T-PLL. ${ }^{4}$ Moreover, T-PLL has a more mature $\mathrm{T}$ cell phenotype expressing CD7, CD2, and CD5 on the cell surface, usually with membrane CD3 and being CD4(+), CD8(-). ${ }^{4}$ In this case only $\mathrm{CD} 7$ and $\mathrm{CCD} 3$ were expressed, suggesting an immature $T$ cell phenotype. This was confirmed by the coexpression of CD34 and CD38. Expression of CD13 and CD33 myeloid associated markers is a relatively common feature in acute lymphoblastic leukaemia blast cells, ${ }^{5}$ being most typical in the most immature cases. ${ }^{6}$ The expression of cCD3 and the absence of reactivity for myeloperoxidase rule out the diagnosis of a myeloid disorder, and this agrees with the morphological findings. In spite of the immature phenotype, Tdt was negative and the disease took a chronic course, which militates against the possibility of a lymphoblastic leukaemia. ${ }^{7}$ The absence of growth after in vitro stimulation with a $\mathrm{T}$ cell mitogen (PHA) could also be related to the abnormal and immature phenotype. This is, in fact, another argument against a T-PLL in which metaphases are normally easily obtained after in vitro stimulation with PHA, and chromosomal abnormalities, often involving the chromosome band 14(11q) are observed. ${ }^{4}$ In spite of the immature phenotype the white cell count rose insidiously during the course of the disease, suggesting a low in vivo proliferative rate, which was confirmed by cell cycle analysis. The absence of enlarged organs could also be related to the immature phenotype and the absence of homing receptors, determining an accumulation in the bone marrow and peripheral blood without gross involvement of other haematopoietic organs.

Another hypothesis for diagnosis is a leukaemia of natural killer-cell lineage. These leukaemias include a heterogenous group of lymphoproliferative disorders with variable clinical manifestations, characterised by the proliferation of "large granular lymphocytes" which have natural killer cell activity, TCR genes in germline configuration, and a heterogenous immunophenotype, the main features being negativity for $\mathrm{CD} 3$ and variable expression of natural killer cell-assocated antigens. ${ }^{8}$ Indeed, this was our first presumed diagnosis, when immunophenotyping showed that the neoplastic clone was strongly positive for CD7 and CD56 and negative for surface CD3, and that the $\beta$ chain TCR gene was in germline configuration. Several other factors, however, argue against this: large granular lymphocytes were never observed, lymphoid cells were positive for $\mathrm{cCD} 3$ and, finally, other natural killer cell associated markers were not expressed.

All these data would support the diagnosis of a $\mathrm{T}$ cell instead of a natural killer cell proliferation; to the best of our knowledge, no case with these characteristics has been reported before.

1 Bennet JM, Catovsky D, Daniel MT, Flandrim G, Galton DAG, Granick HR, et al. Proposed revised criteria for the classification of acute myeloid leukemia: a report of the French-American-British Cooperative Group. Ann Intern Med 1985;103:620-4.

2 Bennet JM, Catovsky D, Daniel MT, Flandrin G, Galton DAG, Gralnick HR, et al. The French-American-British (FAB) Cooperative Group. Proposals for the classification of the chronic (mature) $B$ and $T$ lymphoid leukaemia. $₹$ Clin Pathol 1989;42:567-84.

3 Matutes E, Worner I, Sainati L, de Oliveira MP, Catovsky D. Advances in the lymphoproliferative disorders. Review of our experience in the study of over 1000 cases. Biol Clin Hematol 1989;11:53-62.

4 Matutes E, Brito-Babapulle V, Swansbury J, Ellis J, Morilla R, Dearden C, et al. Clinical and laboratory features of 78 cases of pro-lymphocytic leukemia. Blood 1991;78:3269-74.

5 Drexler HG, Thiel E, Ludwig WD. Review of the incidence and clinical relevance of myeloid antigen-positive

6 Garand R, Voisin S, Papin S, Praloran V, Lenormand B, Favre $M$, et al. Characterization of pro-T ALL subgroups: Comparison with late T-ALL. Leukemia groups: Compa

7 Gomez E, San Miguel JF, Gonzalez M, Orfão A, LopezBerges C, Rios A, et al. Heterogenicity of T cell lymphoblastic leukaemia. $\mathcal{f}$ Clin Pathol 1991;44:628-3

8 Scott CS, Richards SJ, Sivakumaran M, Short M, Child JA, Hunt KM, et al. Transient and persistent expansions of large granular lymphocytes (LGL) and NK-associated (NKa) cells. The Yorkhire Leukaemia Group Study. Br $\mathcal{F}$ Haematol 1993;83:504-15. 